\title{
LESIONES ANÁTOMO-PATOLÓGICAS EN CUYES INTOXICADOS EXPERIMENTALMENTE CON Pteridium aquilinum COMO MODELO ANIMAL PARA BOVINOS CON HEMATURIA VESICAL ENZOÓTICA BOVINA
}

\author{
Anatomo-Pathologycal Lesions in Guinea Pigs Experimentally Intoxicated \\ with Pteridium aquilinum As a Model Animal for Cattle With Bovine \\ ENZOOTIC HAEMATURIA
}

Mariella Ramos G. ${ }^{1,4}$, Alfonso Chavera C. ${ }^{1,5}$, Luis Tabacchi N. ${ }^{1}$, Héctor Huamán U. ${ }^{2, \mathbf{r}}$, Nieves Sandoval C. ${ }^{1}$, José Rodríguez G. ${ }^{3}$

\section{RESUMEN}

\begin{abstract}
El estudio tuvo como objetivo describir el desarrollo de lesiones anátomo-patológicas de cuyes (Cavia porcellus) intoxicados experimentalmente por vía oral con pellets conteniendo Pteridium aquilinum $(\mathrm{Pa})$ por 135 días. El cuy se empleó como modelo experimental para bovinos con Hematuria Vesical Enzoótica Bovina (HVEB). Los pellets contenían un tercio de $\mathrm{Pa}$ y dos tercios de concentrado balanceado para cuyes. Se emplearon 12 cuyes hembras, con peso promedio de $400 \mathrm{~g}$. Se sacrificaron dos individuos al azar a los 30, 60, 90 y 120 días y los 4 restantes cuyes a los 135 días de iniciado el estudio. Las lesiones desarrolladas fueron de tipo neoplásicas, inflamatorias, degenerativas y de procesos de adaptación. Las neoplasias se desarrollaron principalmente en vejiga, pulmón, intestino, bazo y linfonódulos. En la vejiga se desarrollaron neoplasias epiteliales (carcinomas de células transicionales) y no epiteliales (leiomiosarcoma y mixoma), junto con procesos inflamatorios (cistitis no supurativa crónica) y procesos vasculares (telangiectasia y edema suburotelial). En el pulmón, intestino, bazo y linfonódulos, la neoplasia más frecuente fue el linfoma maligno, junto a procesos inflamatorios como bronconeumonías, enteritis y esplenitis. Entre los procesos proliferativos se observó hiperplasia racemosa epitelial intestinal e hiperplasia folicular linfoide en intestino, bazo y linfonódulos. La mayoría de los procesos descritos, incluyendo las neoplasias, se apreciaron desde los 30 días de iniciado el estudio. Se concluye que el cuy fue de utilidad como modelo animal experimental para bovinos con HVEB, al desarrollar lesiones inflamatorias, degenerativas y neoplásicas similares en las vejigas urinarias.
\end{abstract}

Palabras clave: cuy, cobayo, Cavia porcellus, helecho común, Pteridium aquilinum, lesiones, bovino, HVEB

\footnotetext{
${ }^{1}$ Laboratorio de Histología, Embriología y Patología Veterinaria, ${ }^{2}$ Laboratorio de Bioquímica, Nutrición y Alimentación Animal, ${ }^{3}$ Laboratorio de Fisiología Animal, Facultad de Medicina Veterinaria, Universidad Nacional Mayor de San Marcos, Lima

${ }^{4}$ E-mail: mariella_rg@hotmail.com

${ }^{5}$ E-mail: achavera03@gmail.com
} 
The study describes the development of pathological lesions in guinea pigs (Cavia porcellus) experimentally intoxicated through the ingestion of pellets containing one third of Pteridium aquilinum and two thirds of concentrate during 135 days. The guinea pig was used as a experimental model for cattle with Bovine Enzootic Haematuria (BEH). Twelve female animals with a mean of $400 \mathrm{~g}$ body weight were used. Two of them, selected at random, were slaughtered on days 30,60,90 and 120 days and the last 4 on day 135 of the trial. The lesions developed were neoplastic, inflammatory, degenerative and adaptation processes. Tumors mainly developed in the bladder, lung, intestine, spleen and lymph nodes. In the bladder, epithelial neoplasms (transitional cell carcinomas) and nonepithelial neoplasms (leiomyosarcoma and myxoma), together with inflammatory processes (chronic nonsuppurative cystitis) and vascular processes (telangiectasia and edema suburotelial) developed. In the lung, intestine, spleen and lymph nodes, most tumors were malignant lymphoma with inflammatory processes such as bronchopneumonia, enteritis and splenitis. Among the proliferative processes, racemose intestinal epithelial hyperplasia and lymphoid follicular hyperplasia in the intestine, spleen and lymph nodes were observed. Most of the processes including neoplasms were noted as of 30 days. It is concluded that guinea pig can be used as experimental animal model for bovine BEH as develops inflammatory lesions, degenerative, adaptation processes and similar neoplasms in the urinary bladder.

Key words: guinea pig, Cavia porcellus, bracken fern, Pteridium aquilinum, lesions, bovine, $\mathrm{BEH}$

\section{INTRODUCCIÓN}

La Hematuria Vesical Enzoótica Bovina (HVEB) es una enfermedad de distribución mundial y de naturaleza tóxica. Se produce cuando el bovino ingiere regularmente el Pteridium aquilinum $(\mathrm{Pa})$, una planta con características tóxicas, carcinogénicas y mutagénicas, especialmente en la vejiga urinaria (Alonso-Amelot et al., 1993; Oliveira et al., 2006).

En el Perú, la HVEB está presente en el bovino de crianza extensiva en zonas tropicales, siendo la hematuria el principal signo clínico observado. El desarrollo de las lesiones macroscópicas y microscópicas depende de factores tales como la planta, el animal y el medio ambiente. Las lesiones macroscópicas incluyen tumores vesicales de crecimiento exofitico, hemorragias a nivel del urotelio vesical y carcinoma en esófago. Además, disminución de la actividad de la médu- la ósea y depleción linfoide (Marrero et al., 2001). En las lesiones microscópicas de vejiga se describen proliferaciones polipoides y vasculares, procesos inflamatorios como cistitis no supurativa, y procesos neoplásicos como carcinoma, papiloma, hemangioma, hemangiosarcoma y mixosarcoma (Gonzáles et al., 2004).

El inicio de la carcinogenicidad en el urotelio vesical se debe a la transformación del ptaquilósido (principal glucósido carcinógeno) en dienona, en presencia del $\mathrm{pH}$ alcalino de la orina. La dienona es capaz de ocasionar cambios morfológicos progresivos en el urotelio vesical como hiperplasia, papilomas y, finalmente, carcinomas de células transicionales con metaplasia escamosa y glandular (Cohen, 2002).

Esta enfermedad ocasiona serias pérdidas económicas a los ganaderos, además de tener repercusiones en la salud pública. Los ganaderos se ver forzados a beneficiar 
sus animales antes de observar el progreso de la enfermedad; es decir, pérdida de peso, anemia y, finalmente, la muerte. El problema en la salud pública se debe a la toxicidad presente en los subproductos de los bovinos con HVEB como es el caso de la leche que contiene el ptaquilósido, predisponiendo el desarrollo de cáncer gástrico en el humano.

El presente trabajo plantea reproducir la enfermedad del bovino con HVEB usando al cuy como modelo animal, a través del consumo de pellets conteniendo Pa. El cuy, al ser un animal pequeño, de fácil manejo y crianza, con características similares a los bovinos, tales como ser herbívoros y de $\mathrm{pH}$ alcalino en la vejiga, puede favorecer la reproducción de la enfermedad. Además, el desarrollo de neoplasias en un periodo corto y el acceso a los órganos afectados, permite caracterizar las lesiones anátomo-patológicas con respecto a su origen blastodérmico.

\section{MATERIALES y Métodos}

Se colectó helecho común (Pteridium aquilinum), ontogenéticamente juvenil, por la mayor cantidad de compuestos tóxicos en los brotes, en la provincia de Oxapampa, departamento de Cerro de Pasco, entre julio y setiembre de 2008. Los brotes poseen mayor cantidad de compuestos tóxicos y son más digestibles por la escasa lignificación a diferencia de la planta madura (Alonso-Amelot et al., 1999). Una vez hecha la colecta, se secaron bajo sombra por 30 días para preservar su color verde natural y para mantener sus propiedades tóxicas (Pamukcu et al., 1972). Finalmente, fueron empaquetadas en sacos de polietileno y transportadas a Lima en los meses de agosto y octubre para el proceso de peletización en una proporción de 2:1 (dos partes de concentrado balanceado por una de helecho molido).

En el bioterio de la Facultad de Medicina Veterinaria (FMV) de la Universidad Nacional Mayor de San Marcos (UNMSM), se trabajó con 12 cuyes hembras, de $400 \mathrm{~g}$ de peso promedio. Se les proporcionó el alimento pelletizado, en proporción al 10\% del peso vivo hasta por 135 días.

Se sacrificaron dos animales, al azar, a los 30, 60, 90, 120 días de iniciado el estudio, y los 4 restantes a los 135 días. En la eutanasia se utilizó éter como anestésico inhalatorio seguido de degollamiento. En la necropsia, se examinó el intestino, estómago, hígado, bazo, glándulas adrenales, riñón, vejiga, uréter, pulmón, corazón, esófago, tráquea, ovario, cuerno uterino, nódulos linfáticos, vagina y vejiga. Se consideró coloración, tamaño, consistencia, forma, localización y extensión. Órganos y tejidos con lesiones aparentes se colocaron en formol tamponado al 10\%. Las muestras fueron incluidas en parafina, donde se hicieron cortes de $5 \mu \mathrm{m}$ de espesor y se colocaron en láminas portaobjetos. Luego se procedió a su tinción con hematoxilina-eosina (AFIP, 1995) para la lectura histopatológica.

Las necropsias, procesamiento de las muestras y análisis histopatológico, se realizaron en el Laboratorio de Histología, Embriología y Patología Animal de la FMV-UNMSM.

\section{Resultados y Discusión}

Se observó hematuria en tres animales al $4^{\circ}$ día de iniciado el estudio, mientras que en otros reportes con cuyes alimentados con P. aquilinum se describe la ocurrencia de hematuria al $5^{\circ}$ día (Ushijima et al., 1983), y en ratones suizos al $4^{\circ}$ y $5^{\circ}$ día (Pamukcu et al., 1972). Por otro lado, la literatura señala que en bovinos se observa macrohematuria asociado a neoplasias en vejiga, como carcinomas, papilomas y hemangiomas, desde los 335 días, y microhematuria luego de 14 meses de iniciado el estudio (Pamucku y Price, 1969).

En el Cuadro 1, se observan las principales lesiones vasculares encontradas a partir del día 30 de iniciado el estudio. Además, se observó congestión y edema en pulmón, riñón, hígado, vesícula biliar y vejiga, tume- 
Cuadro 1. Lesiones vasculares, degenerativas y procesos de adaptación en cuyes intoxicados experimentalmente con Pteridium aquilinum durante 135 días

\begin{tabular}{|c|c|c|}
\hline $\begin{array}{l}\text { Lesiones vasculares, degenerativas y procesos de } \\
\text { adaptación }\end{array}$ & $\begin{array}{l}\text { Número } \\
\text { de casos }\end{array}$ & $\begin{array}{c}\text { Días de } \\
\text { presentación }^{1}\end{array}$ \\
\hline \multicolumn{3}{|l|}{ Corazón } \\
\hline Hipertrofia muscular & 4 & 90,135 \\
\hline \multicolumn{3}{|l|}{ Hígado } \\
\hline Hepatosis & 10 & $30,60,90,120,135$ \\
\hline Hipertrofia muscular arteriolar portal & 2 & 120,135 \\
\hline Hiperplasia de los conductos biliares & 1 & 135 \\
\hline \multicolumn{3}{|l|}{ 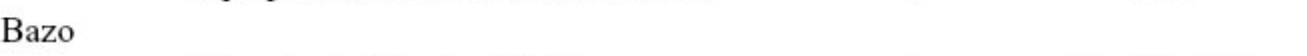 } \\
\hline Hiperplasia folicular linfoide & 3 & $90,120,135$ \\
\hline \multicolumn{3}{|l|}{ Pulmón } \\
\hline $\begin{array}{l}\text { Bronquiolisis, hipertrofia muscular } \\
\text { arteriolar pulmonar }\end{array}$ & 1 & 30 \\
\hline \multicolumn{3}{|l|}{ Esófago } \\
\hline Esofagopatía bacteriana & 1 & 60 \\
\hline Esofagopatía micótica & 2 & 60,135 \\
\hline \multicolumn{3}{|l|}{ Intestino } \\
\hline Hiperplasia racimosa epitelial & 15 & $30,60,90,120,135$ \\
\hline Hiperplasia folicular linfoide & 4 & $30,90,135$ \\
\hline \multicolumn{3}{|l|}{ Vesícula biliar } \\
\hline $\begin{array}{l}\text { Telangiectasia, linfagiectasia y edema } \\
\text { subepitelial }\end{array}$ & 3 & $30,60,135$ \\
\hline \multicolumn{3}{|l|}{$x_{\mathrm{P}}$} \\
\hline $\begin{array}{l}\text { Telangiectasia, linfagiectasia, edema } \\
\text { suburotelial }\end{array}$ & 12 & $30,60,90,120,135$ \\
\hline
\end{tabular}

facción turbia en los hepatocitos y túbulos renales, y hemorragias petequiales en la superficie del urotelio. Similares cambios vasculares fueron reportados en ratones y cobayos intoxicados con $\mathrm{Pa}$ a partir del día 10 de iniciado el estudio por Pamukcu et al. (1972) y Ushijima et al. (1983). Entre las lesiones degenerativas y proliferativas destaca la proliferación de conductos biliares y cambios proliferativos a nivel intestinal, lesiones que han sido reportadas en cuyes entre los 31 y 54 meses de iniciado el estudio (Dawra et al., 2002).
El Cuadro 2 presenta las lesiones inflamatorias, a veces acompañadas con lesiones neoplásicas descritas en el Cuadro 3. Por ejemplo, la presencia de carcinomas en vejigas y células mononucleares de distribución difusa y en agregados linfoides a nivel del suburotelio (Fig. 1B) correspondió a cistitis no supurativa junto a neoplasias. Se menciona que estos infiltrados mononucleares están compuestos por linfocitos $T$, células naturales (NK) y macrófagos (Ushijima et al., 1983; Abbas et al., 2002), observados en bovinos con HVEB (Vargas et al., 2003). Además, la reacción inflamatoria es considerada el inicio 
Cuadro 2. Lesiones inflamatorias en cuyes intoxicados experimentalmente con Pteridium aquilinum, durante 135 días

\begin{tabular}{|c|c|c|c|}
\hline \multicolumn{2}{|c|}{ Lesiones inflamatorias } & $\begin{array}{l}\text { Número } \\
\text { de casos }\end{array}$ & $\begin{array}{l}\text { Días de } \\
\text { presentación }^{1}\end{array}$ \\
\hline \multicolumn{4}{|l|}{ Tráquea } \\
\hline & Traqueitis no supurativa & 2 & 135 \\
\hline & Traqueitis eosinofilica & 1 & 135 \\
\hline \multicolumn{4}{|l|}{ Bazo } \\
\hline & Esplenitis supurativa y depleción linfoide & 2 & 30 \\
\hline \multicolumn{4}{|c|}{$\mathrm{P}_{\mathrm{P}}$} \\
\hline & Neumonía intersticial supurativa & 1 & 30 \\
\hline & Neumonía no supurativa hemorrágica & 1 & 90 \\
\hline & Bronconeumonía catarral supurativa & 1 & 60 \\
\hline & $\begin{array}{l}\text { Bronconeumonía catarral no supurativa, } \\
\text { bronquiolisis, HMAP }\end{array}$ & 5 & $30,60,90,120,135$ \\
\hline \multicolumn{4}{|c|}{ Estómago } \\
\hline & Gastritis catarral multifocal aguda & 7 & $30,60,90,120,135$ \\
\hline \multicolumn{4}{|l|}{ Intestino } \\
\hline & Enteritis no supurativa & 4 & $30,60,135$ \\
\hline & $\begin{array}{l}\text { Enteritis linfoplasmocítica asociado a } \\
\text { Balantidium spp }\end{array}$ & 4 & 90 \\
\hline & $\begin{array}{l}\text { Enteritis linfoplasmocítica difusa crónica } \\
\text { asociado a parásitos y bacterias }\end{array}$ & 1 & \\
\hline & Enteritis plasmocítica asociado a bacterias & 4 & 135 \\
\hline \multicolumn{4}{|c|}{ Vesícula biliar } \\
\hline & Colecistitis linfoplasmocítica & 1 & 30 \\
\hline \multicolumn{4}{|l|}{ Riñón } \\
\hline & Glomerulolitis e hiperplasia tubular renal & 1 & 60 \\
\hline & $\begin{array}{l}\text { Glomerulolitis hemorragia medular y } \\
\text { degeneración vacuolar tubular }\end{array}$ & 1 & 90 \\
\hline & $\begin{array}{l}\text { Glomerulitis linfoplasmocítica subaguda } \\
\text { con quistes de retención }\end{array}$ & 2 & 90,135 \\
\hline \multicolumn{4}{|l|}{ Vejiga } \\
\hline & Cistitis no supurativa & 6 & $30,60,90,120$ \\
\hline & Cistitis crónica polipoide & 1 & 135 \\
\hline
\end{tabular}

${ }^{1}$ Los muestreos se realizaron los dias 30, 60, 90, 120 y 135 de iniciado el proceso de intoxicación

de procesos proliferativos y neoplásicos en el urotelio vesical, en tanto que la cistitis se considera el inicio de la carcinogenicidad (Sardon et al., 2005), tal como podría suceder en el caso del linfoma maligno desarro- llado en los cuyes a partir del día 30 del estudio. En ratones suizos se reportó leucemia en bazo y nódulos linfáticos e hiperplasia folicular a partir de las 60 semanas (Pamukcu et al., 1972). 
Cuadro 3. Neoplasias en cuyes intoxicados experimentalmente con Pteridium aquilinum durante 135 días, en base a análisis histopatológicos

\begin{tabular}{|c|c|c|}
\hline Tipo de neoplasia & $\begin{array}{l}\text { Número de } \\
\text { casos }\end{array}$ & $\begin{array}{c}\text { Días de } \\
\text { presentación }^{1}\end{array}$ \\
\hline \multicolumn{3}{|l|}{ Vejiga urinaria } \\
\hline Leiomiosarcoma & 2 & 30 \\
\hline Mixoma & 2 & 30 \\
\hline $\mathrm{CCT}^{2}$ infiltrativo y papilar & 5 & $30,60,135$ \\
\hline CCT infiltrativo y no papilar & 4 & $90,120,135$ \\
\hline CCT no infiltrativo y papilar & 2 & 120,135 \\
\hline \multicolumn{3}{|l|}{ Bazo } \\
\hline Linfoma maligno & 4 & $60,90,120,135$ \\
\hline \multicolumn{3}{|l|}{ Pulmón } \\
\hline Carcinoma pulmonar & 1 & 30 \\
\hline Linfoma & 3 & $90,120,135$ \\
\hline \multicolumn{3}{|l|}{ Intestino } \\
\hline Linfoma maligno folicular linfoide & 10 & $30,60,90,135$ \\
\hline \multicolumn{3}{|l|}{ Linfonódulos } \\
\hline Linfoma maligno folicular linfoide & 5 & $30,60,135$ \\
\hline \multicolumn{3}{|l|}{ Vagina } \\
\hline Carcinoma in situ & 1 & 90 \\
\hline Leiomioma & 1 & 90 \\
\hline Carcinoma infiltrativo & 1 & 120 \\
\hline \multicolumn{3}{|l|}{ Vesícula biliar } \\
\hline Carcinoma infiltrativo y papilar & 1 & 135 \\
\hline \multicolumn{3}{|l|}{ (1) } \\
\hline Leiomiosarcoma & 1 & 90 \\
\hline
\end{tabular}

El origen de los procesos neoplásicos e inflamatorios se relacionan con la actividad inmunosupresora del helecho común, ocasionando depleción linfoide esplénica e inhibiendo la inmunidad celular (linfocitos T y NK), principales responsables de la inmunidad antitumoral (Oliveira et al., 2009). Además, la actividad carcinogénica de la dienona en presencia de $\mathrm{pH}$ alcalino en los múltiples órganos del cuy, como el caso de la orina sobre el urotelio.

En el Cuadro 3, se muestran los tejidos neoplásicos encontrados en el estudio. La neoplasia epitelial más frecuente en vejiga fue el carcinoma de células transicionales (CCT) (Fig. 1A), y entre las neoplasias mesenquimales, el leiomiosarcoma (Fig. 1C) y el mixoma (Fig. 1D). En pulmón, intestino, bazo y linfonódulos se desarrolló linfoma maligno (Fig. 2) con procesos inflamatorios tales como bronconeumonías, enteritis y esplenitis.

Se plantea que el desarrollo de los procesos inflamatorios, proliferativos, degenerativos y neoplásicos encontrados en el presente estudio y los mencionados en la discusión, se deben a múltiples factores relacionados a la 

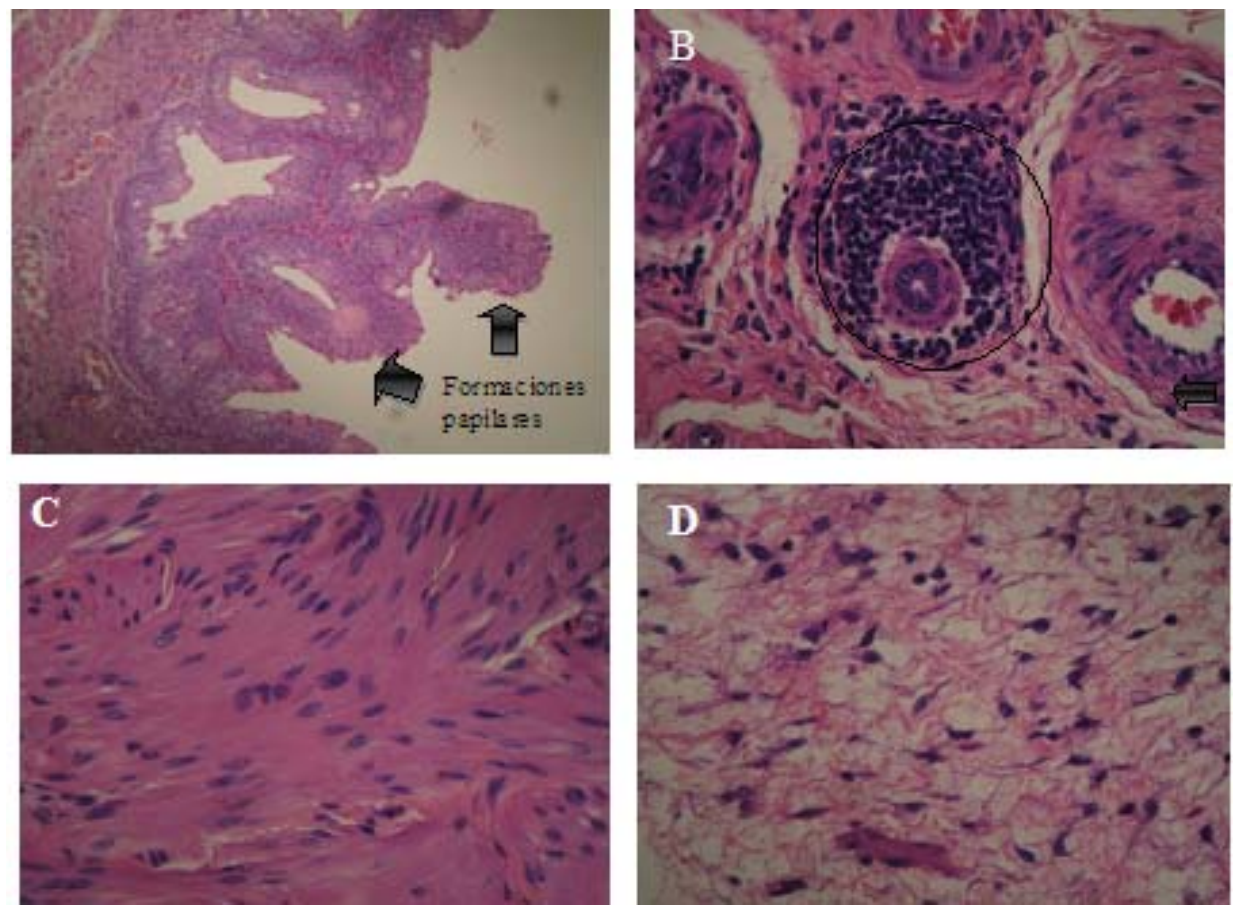

Figura 1. Vejiga de cuy a los 30 días de iniciado el proceso de intoxicación con Pteridium aquilinum (Tinción Hematoxilina-Eosina). A: Carcinoma de células transicionales (CCT) y las formaciones papilares uroteliales (100x); B: Perivasculitis linfocítica en el suburotelio (400x); C: Leiomiosarcoma (400x); D: Mixoma (400x)
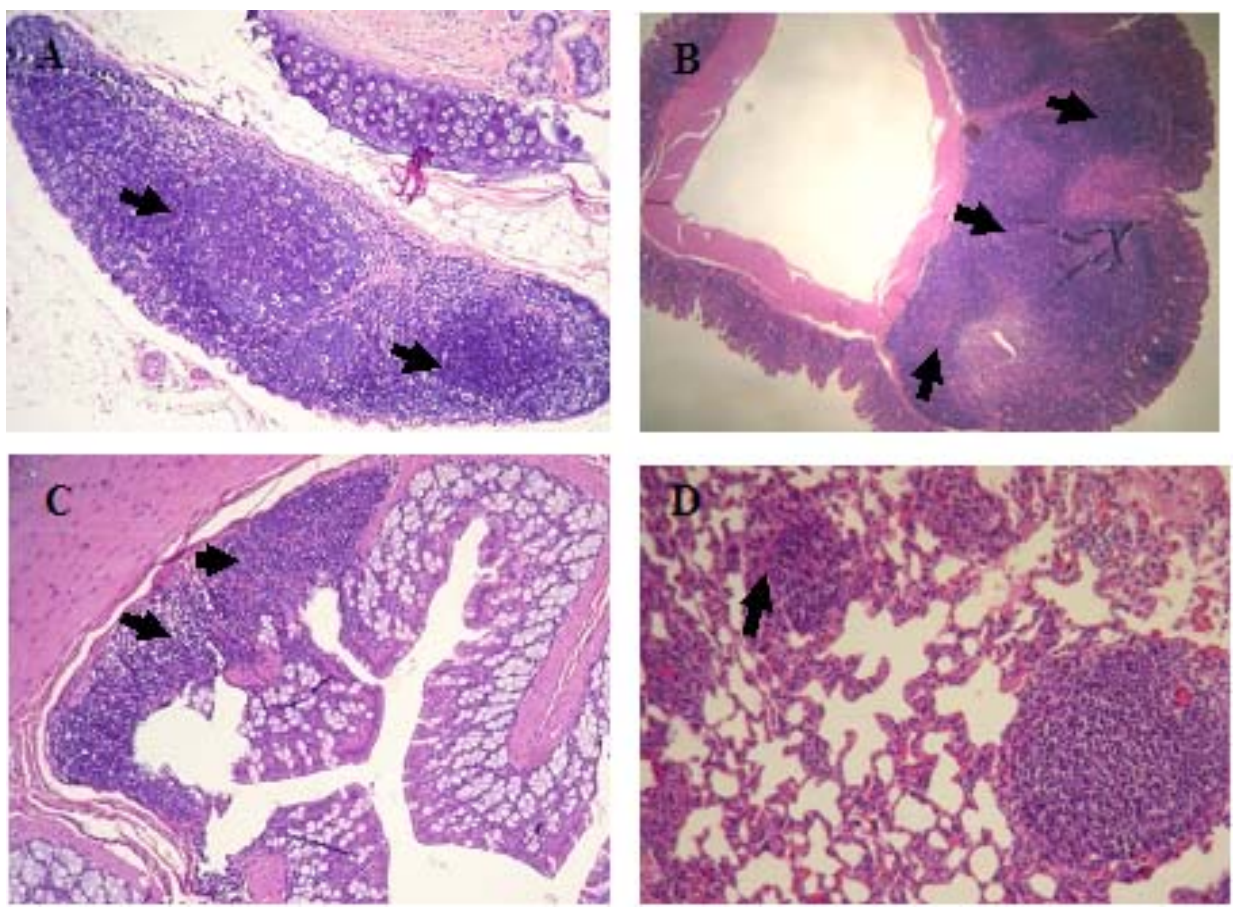

Figura 2. Linfoma maligno en cuyes intoxicados experimentalmente con Pteridium aquilinum (Tinción Hematoxilina-Eosina). A: Linfoma maligno linfonodular peritraqueal (40x); B: Linfoma maligno folicular linfoide intestinal (100x); C: Linfoma maligno en colon (100x); D: Linfoma pulmonar con múltiples formaciones foliculares compactas de linfocitos y moderada bronconeumonía mucocatarral linfoplasmocítica difusa crónica (100x) 
planta, animal y medio ambiente. En los estudios donde se empleó P. aquilinum joven, se desarrollaron neoplasias en un periodo más corto. Además, es posible que los niveles de toxicidad varíen según los ecotipos del Pa en el mundo.

\section{Conclusiones}

- Las lesiones histopatológicas y aparición de procesos neoplásicos en el cuy por efecto de la intoxicación experimental con Pteridium aquilinum se apreciaron desde la primera necropsia (30 días de iniciado el estudio).

- Las lesiones observadas en el cuy fueron muy similares a las reportadas en bovinos.

- El cobayo podría ser utilizado como modelo animal experimental para bovinos con Hematuria Vesical Enzoótica Bovina.

\section{Literatura Citada}

1. Abbas S, Lichtman A, Pober J. 2002. Inmunología antitumoral. En: Abbas $\mathrm{S}$, Lichtman A, Pober J (eds). Inmunología celular y molecular. $4^{\text {a }}$ ed. Madrid: Mc Graw-Hill. p 398-418.

2. [AFIP] Instituto de Patología de las Fuerzas Armadas de los Estados Unidos de América. 1995. Métodos histotecnológicos. Washington DC: AFIP. $279 \mathrm{p}$.

3. Alonso-Amelot ME, Castillo U, Jongh F. 1993. On the passage of the Bracken fern carcinogen, ptaquilósido into bovine milk. Le Lait 73: 323-332.

4. Cohen SM. 2002. Comparative pathology of proliferative lesions of the urinary bladder. Toxicol Pathol 30: 663-671.

5. Dawra RK, Kurade NP, Sharma OP. 2002. Carcinogenicity of the fern Pteridium aquilinum collected from enzootic bovine haematuria-free hilly area in India. Current Sci 83: 1005-1009.
6. Gonzáles C, Chavera A, Perales $R$, Falcón N, Evaristo R. 2004. Caracterización de las lesiones encontradas en bovinos con hematuria vesical enzoótica en la zona de Oxapampa, Pasco. Rev Inv Vet, Perú 15: 25-36.

7. Marrero E, Bulnes C, Sánchez LM, Palenzuela I, Stuart R, Jacobs F, Romero J. 2001. Caracterización general de cuadro de toxicosis por P. aquilinum en bovinos en el departamento de Tarija, Bolivia. Rev Salud Anim 23(1): 16-22.

8. Oliveira PA, Colaco A, de la Cruz LF, Lopes C. 2006. Experimental bladder carcinogenesis-rodent models. Exp Oncol 28(1): 2-11.

9. Oliveira A, Furlan MS, Sakai M, Fukumasu H, Machado I, Haraguchi M, Lima S. 2009. Immunomodulatory effects on Pteridium aquilinum on natural killer activity and select aspects of the cellular immune response of mice. J Immunotoxicol 6: 104-114.

10. Pamukcu AM, Erturk E, Price JM, Bryan GT. 1972. Lymphatic leukemia and pulmonary tumors in female swiss mice fed Bracken fern (Pteris aquiline). Cancer Res 35: 1442-1445.

11. Pamukcu AM, Price JM. 1969. Induction of intestinal and urinary bladder cancer in rats by feeding bracken fern (Pteris aquilina). In: J Natl Cancer Inst 43: 275-279.

12. Sardon D, De la Fuente I, Calonge E, Perez-Alenza M, Castaño M, Dunner S, Peña L. 2005. H-ras immunohistochemical expression and molecular analysis of urinary bladder lesions in grazing adult cattle exposed to bracken fern. J Comp Pathol 132: 195-201.

13. Ushijima J, Matsukawa K, Yuasa A, Okada M. 1983. Toxicities of Bracken fern in guinea pigs. Jpn J Vet Sci 45: 593-602.

14. Vargas P, Nascimento T, Barros C, Hubinger C.2003. Histopathological aspects of Bovine Enzootic Hematuria in Brazil. Pesq Vet Bras 23(2): 65-81. 